\title{
Original
}

\section{Suppression of parotid acinar cell dysfunction by the free radical scavenger 3-methyl-1-phenyl-2-pyrazolin-5-one}

\author{
Hajime Sakurai1), Megumi Yokoyama ${ }^{2,3)}$, Osamu Katsumata-Kato2,3), \\ and Junko Fujita-Yoshigaki²,3)
}

\begin{abstract}
1)Division of Cellular Physiology, Nihon University Graduate School of Dentistry at Matsudo, Matsudo, Japan 2)Department of Physiology, Nihon University School of Dentistry at Matsudo, Matsudo, Japan 3)Research Institute of Oral Science, Nihon University School of Dentistry at Matsudo, Matsudo, Japan
\end{abstract}

(Received November 7, 2018; Accepted December 11, 2018)

\begin{abstract}
Salivary gland atrophy and consequent hyposalivation are serious problems in clinical dentistry, as saliva regulates the environment of the oral cavity. To clarify the mechanisms underlying salivary gland dysfunction, a system for primary culture of parotid acinar cells has been established. It has been reported previously that the process of cell isolation from parotid glands triggers stress signaling mediated by Src and p38 mitogen-activated protein (MAP) kinase (p38), leading to dedifferentiation of acinar cells, and that an nicotinamide adenine dinucleotide phosphate (NADPH) oxidase inhibitor suppresses this activation of Sre and p38, suggesting that reactive oxygen species initiate the dedifferentiation signal. The present study examined the effect of a free radical scavenger, 3-methyl-1-phenyl-2-pyrazolin-5-one (also termed MCI-186 or edaravone), on activation of the stress signal and the secretory function of parotid acinar cells. Activation of $\mathrm{p} 38$ during cell isolation was suppressed by addition of MCI-186. The retention of the activity of amylase, a major salivary protein, and the number of amylase-containing secretory granules were improved by isolation and culture in the presence of MCI-186. In addition, calcium elevation upon stimulation with a muscarinic agonist was
\end{abstract}

Correspondence to Dr. Junko Fujita-Yoshigaki, Department of Physiology, Nihon University School of Dentistry at Matsudo, 2-870-1 Sakaecho-nishi, Matsudo, Chiba 271-8587, Japan Fax: +81-47-360-9327 E-mail: yoshigaki.junko@nihon-u.ac.jp

J-STAGE Advance Publication: August 3, 2019

Color figures can be viewed in the online issue at J-STAGE.

doi.org/10.2334/josnusd.18-0405

DN/JST.JSTAGE/josnusd/18-0405 higher in MCI-186-treated cells than in untreated cells. These results suggest that MCI-186 (edaravone) is a promising agent for prevention of salivary gland dysfunction.

Keywords: salivary glands; dry mouth; hyposalivation; oxidative stress; calcium signaling; exocrine.

\section{Introduction}

Saliva is essential for maintenance of the oral environment. Hyposecretion of saliva causes serious caries, periodontitis, and oral mucosal infections due to poor oral hygiene (1). In addition, the associated decrease in salivary proteins, which have lubrication properties, also impairs mastication, swallowing and articulation. Therapeutic radiation for head and neck cancers causes apoptosis and atrophy of acinar cells and an increase of duct cells and connective tissues in salivary glands. Thus, many such patients suffer an irreversible decrease in saliva after radiotherapy. In addition, chronic inflammatory conditions, such as Sjögren's syndrome, can induce tissue damage and decrease parenchymal cells. Therefore, approaches for protecting salivary gland cells from such damage are urgently needed.

It is difficult to maintain the functions of exocrine cells, such as salivary acinar cells, in primary cultures or as cell lines. This is one of the major problems hindering studies of salivary acinar cell function. To study the mechanisms allowing maintenance of salivary acinar cell secretory function, a system for primary culture of parotid acinar cells that maintain their capacity to generate secretory granules (SGs) and stimulus-dependent secretion of sali- 
vary proteins has been established (2). However, it was found that this capacity gradually decreased, and that expression of differentiation markers changed during culture (3); the expression levels of acinar markers, such as amylase and aquaporin-5, decreased, while ductal markers and mesenchymal markers increased. Inhibitors of Src and p38 mitogen-activated protein (MAP) kinase (p38) suppressed these changes and increased the expression of acinar marker proteins. These results suggest that the Src-p38 signaling pathway plays a role in triggering cell dedifferentiation to ensure survival in response to cellular stress (4). Diphenyleneiodonium (DPI), nicotinamide adenine dinucleotide phosphate (NADP) oxidase inhibitor, suppresses activation of Src and p38 (5). Therefore, reactive oxygen species (ROS) may be generated during cell isolation, and these may activate Src. It has been suggested that oxidative stress is involved in the development of Sjögren's syndrome (6) and hyposalivation after $\gamma$-irradiation (7).

However, the use of DPI to suppress stress signals carries a risk of side effects, because DPI also has inhibitory activities against other enzymes, such as nitric oxide synthetases. The present study was performed to examine the effect of 3-methyl-1-phenyl-2-pyrazolin5-one (also known as MCI-186 or edaravone), which has been approved for the treatment of acute-phase brain infarction and amyotrophic lateral sclerosis $(8,9)$, on the dedifferentiation signaling and secretory function of parotid acinar cells.

\section{Materials and Methods}

\section{Materials}

Rabbit polyclonal anti-amylase and mouse monoclonal anti-occludin antibodies were purchased from Merck (Darmstadt, Germany). Mouse monoclonal anti-p38 and rabbit polyclonal anti-phosphorylated p38 antibodies were purchased from BD Biosciences (Sparks, MD, USA) and R\&D Systems (Minneapolis, MN, USA), respectively. MCI-186 was purchased from Cayman Chemical Company (Ann Arbor, MI, USA). Culture medium and supplements were purchased from Thermo Fisher Scientific (Waltham, MA, USA).

\section{Preparation and culture of isolated acinar cells}

All animal experiments were carried out in accordance with institutional and national guidelines for the care and use of experimental animals and were approved by the Experimental Animal Committee of the Nihon University School of Dentistry at Matsudo (AP15MD018). Parotid glands were removed from male Sprague-Dawley rats (weighing 150-200 g) under inhalation anesthesia with $3 \%$ sevoflurane. Acinar cells were isolated from the glands as described previously (10). Briefly, finely minced glands were digested with collagenase and hyaluronidase in isolation buffer (Hanks' balanced salt solution containing $20 \mathrm{mM}$ HEPES-NaOH, $\mathrm{pH} 7.4$ ) in the absence or presence of MCI-186. The isolated cells were diluted to $0.3 \mathrm{mg} / \mathrm{mL}$ protein in Waymouth's medium containing $10 \%$ rat serum, ITS-X supplement, $1 \mu \mathrm{M}$ hydrocortisone, $100 \mathrm{U} / \mathrm{mL}$ penicillin, $0.1 \mathrm{mg} / \mathrm{mL}$ streptomycin and $10 \mathrm{nM}$ cystatin, and cultured at $37^{\circ} \mathrm{C}$ in $5 \%$ $\mathrm{CO}_{2}$. The medium was changed 1 day after cell isolation. MCI-186 was dissolved in dimethylsulfoxide (DMSO) to a concentration of $50 \mathrm{mM}$ as a stock solution, and then diluted 1:1,000 to a final concentration of $50 \mu \mathrm{M}$ for use. The same volume of DMSO was added to the control medium (final, $0.1 \%$ ).

\section{SDS-PAGE and immunoblotting analysis}

Cells were lysed with $20 \mathrm{mM}$ HEPES-NaOH (pH7.4) containing $0.1 \%$ Triton X-100 and $1 \times$ Complete Protease Inhibitor Cocktail (Roche, Basel, Switzerland). The proteins were separated by SDS-PAGE and transferred to Hybond-LFP membranes (GE Healthcare, Chicago, IL, USA). The membranes were blocked at room temperature for $1 \mathrm{~h}$ in blocking reagent (GE Healthcare) and probed with the primary antibodies. Immunoreactivity was determined using ECL-Plex (GE Healthcare), and images were acquired using Typhoon Trio (GE Healthcare). The intensities of the immunoreactivities were quantified using ImageQuantTL software (GE Healthcare). The intensity of immunoreactivity in each MCI-treated sample was normalized to that in an untreated sample on the same blotting membrane. After the normalized values of total and phosphorylated p 38 had been acquired, the ratio of the phosphorylated form to total p38 was calculated in each experiment.

\section{Immunofluorescence microscopy of cultured acinar cells}

Acinar cells were plated on collagen I-coated dishes (Iwaki, Tokyo, Japan) and cultured for 3 days. The cells were then treated with $10 \%$ formalin in phosphate-buffered saline (PBS) for $5 \mathrm{~min}$ and subsequently treated with $0.2 \%$ Triton X-100 in PBS for 10 min. After blocking with bovine serum albumin and preimmune goat IgG, the cells were labeled with the primary antibodies, followed by the respective Alexa Fluor-labeled secondary antibodies. Images were acquired using a $100 \times 1.46$ $\alpha$ Plan-Apochromat oil objective lens on an LSM-510 laser confocal microscope (Carl Zeiss, Oberkochen, Germany). Optical sections were taken along the z-axis 
at $0.5-\mu \mathrm{m}$ intervals. The number of granules was counted by spot analysis with Imaris software (Bitplane, Zurich, Switzerland). Amylase-positive spots were selected with a threshold diameter of $0.5 \mu \mathrm{m}$. When the software did not count the spots because of overcrowding, they were excluded because it was not possible to judge whether they were granules.

\section{Amylase release assay}

Cells cultured in dishes were incubated with release buffer (20 mM HEPES-NaOH, pH 7.4, $128 \mathrm{mM} \mathrm{NaCl}, 4$ $\mathrm{mM} \mathrm{KCl}, 1.2 \mathrm{mM} \mathrm{MgCl}_{2}, 1 \mathrm{mM} \mathrm{CaCl}_{2}, 1 \mathrm{mM} \mathrm{NaH}_{2} \mathrm{PO}_{4}$, $5 \mathrm{mM}$ hydoxybutyrate) in the absence or presence of $10^{-6}$ $\mathrm{M}$ isoproterenol (Iso) at $37^{\circ} \mathrm{C}$ for $15 \mathrm{~min}$. After incubation, the solution was removed, and the remaining cells were harvested. Amylase activity was measured using a method reported previously (2). One unit of amylase was defined as the quantity of enzyme that liberates $1 \mathrm{mg}$ of maltose during $1 \mathrm{~min}$ at $30^{\circ} \mathrm{C}$.

\section{Measurement of intracellular $\mathrm{Ca}^{2+}$ mobilization}

Isolated parotid acinar cells were loaded with $2 \mu \mathrm{M}$ fura-2 AM (Thermo Fisher) in loading buffer (10 mM HEPES$\mathrm{NaOH}, \mathrm{pH} 7.4,140 \mathrm{mM} \mathrm{NaCl}, 5 \mathrm{mM} \mathrm{KCl}, 1 \mathrm{mM} \mathrm{MgCl}_{2}$, $2.5 \mathrm{mM} \mathrm{CaCl}_{2}, 10 \mathrm{mM}$ glucose, $1.25 \mathrm{mM}$ probenecid, and $0.2 \%$ bovine serum albumin) at $37^{\circ} \mathrm{C}$ for $30 \mathrm{~min}$. The cells were rinsed three times and suspended in $\mathrm{Ca}^{2+}$-free buffer. The fluorescence of the fura-2-loaded cells was measured by using CAF-110 spectrofluorometer (Jasco, Tokyo, Japan) with excitation at 340 and $380 \mathrm{~nm}$, and emission at $510 \mathrm{~nm}$. Elevation of the intracellular $\mathrm{Ca}^{2+}$ concentration was evaluated as the increase in the ratio of fluorescence intensities at $340 \mathrm{~nm}$ and $380 \mathrm{~nm}$ (F340/ F380).

\section{Statistical analyses}

All values are reported as means \pm SEM. Statistical analyses of the differences in the means of experimental pairs were evaluated by the paired $t$-test. The $P$ values obtained are indicated in the figure legends when statistically significant.

\section{Results}

\section{Activation of p38 during cell isolation is suppressed by addition of MCI-186}

It had been shown previously that p38 was activated during cell isolation from parotid glands, detected as an increase in its phosphorylation (4). Here, acinar cells were isolated from rat parotid glands in the absence and presence of MCI-186. Phosphorylation of p38 was detected by immunoblotting in the cells that had been
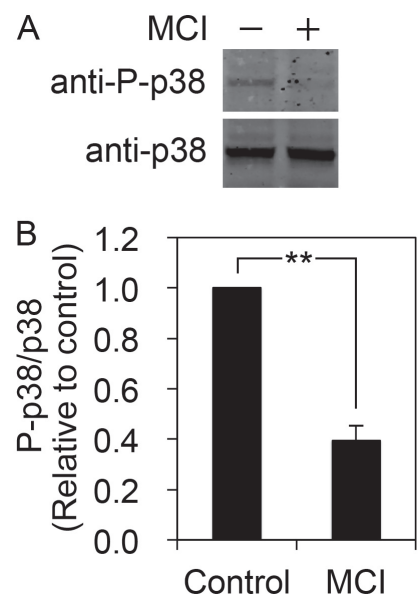

Fig. 1 Suppression of p38 activation by addition of MCI-186. (A) Immunoblotting analysis of lysates from control (-) and MCI186-treated $(+)$ cells with antibodies against the phosphorylated form (anti-P-p38) and total p38 (anti-p38). (B) Quantitative analysis of the immunoblots. After the intensity of immunoreactivity in the MCI-treated sample had been normalized to that in an untreated sample (control) on the same blotting membrane, the ratio of the phosphorylated form to total p38 (P-p38/p38) was calculated in each experiment. Phosphorylation of p38 was significantly suppressed by the addition of MCI-186 $(* * P<0.01$, paired $t$-test, $n=4$ ).

isolated without MCI-186 (Fig. 1). In cells that had been isolated in the presence of MCI-186, however, the phosphorylated p 38 was significantly decreased, although the amount of total p38 was unchanged.

\section{Maintenance of amylase activity and secretory granules in MCI-186-treated cells}

The activity of amylase, the most abundant salivary protein in parotid acinar cells, was examined to evaluate the degree of cell differentiation. After 3 days of culture in the absence and presence of MCI-186, the cells were harvested and their amylase activity was measured as the relative activity per mg of protein in the cell lysate. The relative amylase activity in the MCI-186-treated cells was significantly higher (1.5-fold) than that in the control cells (Fig. 2).

Salivary proteins like amylase are stored in SGs until stimulation. SGs are released through secretagoguedependent exocytosis, which is an important function of exocrine cells. In primary culture, the acinar cells spread over the dish surface and formed a monolayer, and the number of SGs decreased gradually during culture (3). When SGs were labeled with an anti-amylase antibody at 3 days after isolation, the distribution of granules in the cells was not uniform (Fig. 3A); some cells lost most of their granules, whereas others retained many. To compare the number of remaining granules in the control 


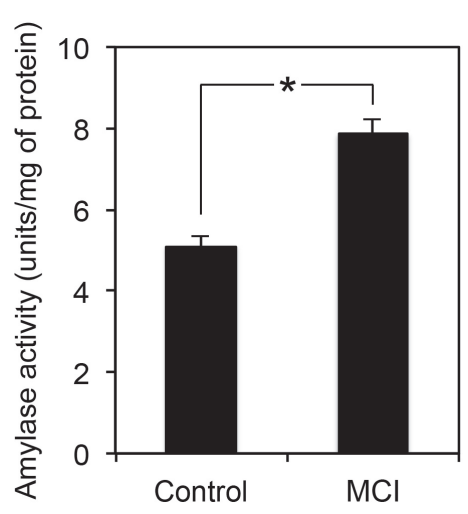

Fig. 2 Effect of MCI-186 on the decrease in relative amylase activity during culture.

The relative amylase activity (units/mg protein) in the lysates from control and MCI-186-treated cells after 3 days of culture is shown. Although the relative amylase activity was lower than that in the cells just after isolation, the addition of MCI-186 suppressed the decrease in amylase activity $(* P<0.05$, paired $t$-test, $n=4)$.

and MCI-186-treated cells (Fig. 3A, B), all the granules were counted in images $(142.86 \mu \mathrm{m} \times 142.86 \mu \mathrm{m})$ of the confluent monolayer, and the number of granules per unit area was calculated. MCI-186-treated cells retained more granules (1.7-fold) than the control cells (Fig. 3C). The difference in the number of granules between the two cultures was consistent with the remaining amylase activity.

\section{Maintenance of stimulus-dependent amylase secretion in both control and MCI-186-treated cells}

The cells were cultured in the absence and presence of isoproterenol (Iso) and the medium was collected for measurement of the released amylase activity, determined as the percentage of the total activity in whole cells. A small amount of amylase was released without stimulation, and Iso enhanced the release from both control and MCI-186-treated cells (Fig. 4). Addition of Iso increased amylase secretion in control and MCI-168-treated cells 2.8-fold and 3.0-fold, respectively. This enhancement was comparable to that observed in the cells just after isolation (2). Stimulus-dependent exocytosis capacity was not greatly impaired, even after 3 days of culture, although the synthesis of the amylase protein declined. MCI-168 did not promote the response to secretagogues, although it increased the number of remaining granules.

\section{Enhancement of the increase in intracellular $\mathrm{Ca}^{2+}$ concentration in MCI-186-treated cells}

Muscarinic stimulation increases the intracellular $\mathrm{Ca}^{2+}$ concentration $\left(\left[\mathrm{Ca}^{2+}\right]_{\mathrm{i}}\right)$, which is essential for fluid secretion of saliva. Control cells isolated without MCI-186 responded to $10 \mu \mathrm{M}$ carbachol $(\mathrm{CCh})$. The rapid increase
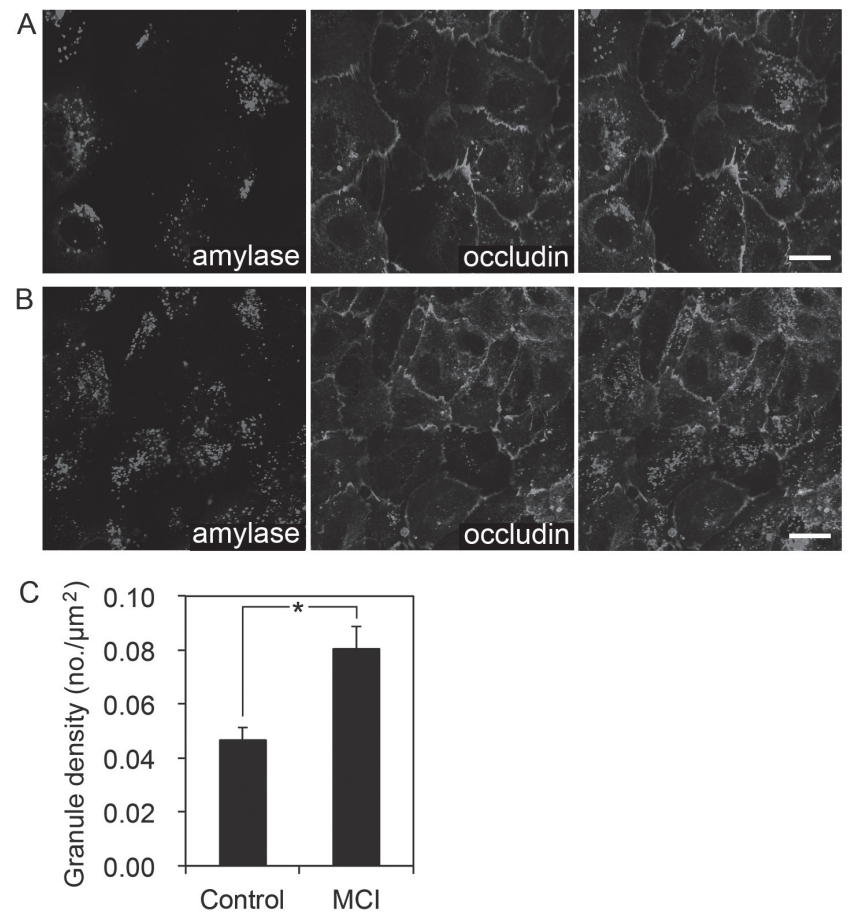

Fig. 3 Effect of MCI-186 on the number of secretory granules retained in cultured acinar cells.

Immunofluorescence microscopy of control (A) and MCI186-treated (B) cells labeled with anti-amylase (red) and anti-occludin (green) antibodies. Optical sections were taken along the $z$-axis at $0.5-\mu \mathrm{m}$ intervals, and the images shown are single z-planes. Spots labeled with the anti-amylase antibody correspond to secretory granules (SGs). Bars, $20 \mu \mathrm{m}$. (C) Quantitative analysis of the density of SGs in the cells. Values are the number of granules per unit area. MCI-186-treated cells retained significantly more granules than the control cells $\left({ }^{*} P<0.05\right.$, $t$-test, $n=8$ ).

in $\left[\mathrm{Ca}^{2+}\right]_{\mathrm{i}}$ observed in the absence of extracellular $\mathrm{Ca}^{2+}$ indicates the release of $\mathrm{Ca}^{2+}$ from the intracellular $\mathrm{Ca}^{2+}$ pool (Fig. 5A). Addition of extracellular $\mathrm{Ca}^{2+}$ (final concentration $2.5 \mathrm{mM}$ ) induced an additional and sustained increase in $\left[\mathrm{Ca}^{2+}\right]_{i}$, corresponding to $\mathrm{Ca}^{2+}$ influx from the extracellular buffer. The CCh-dependent increase in $\left[\mathrm{Ca}^{2+}\right]_{i}$ was higher in MCI-186-treated cells than in control cells (Fig. 5A). The increase from the baseline to the peak F340/F380 was significantly higher in MCI-treated cells than in control cells (Fig. 5B).

\section{Discussion}

MCI-186 is a free radical scavenger widely used to treat ischemic brain damage and neurodegenerative diseases $(8,9)$. It is also thought to protect other organ systems such as the cardiovascular system, and to suppress autoimmune disease (11-13). The present study demonstrated that MCI-186 slightly improved the function of isolated salivary acinar cells.

MCI-186 treatment suppressed the phosphorylation 


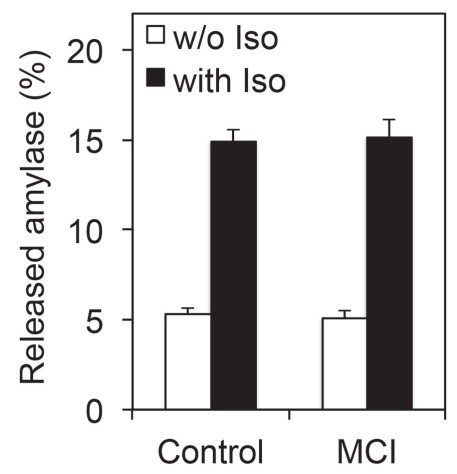

Fig. 4 Response of cultured acinar cells to $\beta$-adrenergic stimulation.

At 3 days after cell isolation, cultured acinar cells were incubated with or without (w/o) $10^{-6} \mathrm{M}$ isoproterenol (Iso) at $37^{\circ} \mathrm{C}$ for 15 $\mathrm{min}$. Amylase activity in the medium and cell lysate was measured, and the amount of released amylase is shown as a percentage of the total amylase. There was no significant difference between the control and MCI-186-treated cells $(n=4$, paired $t$-test).

of p38, which is mediated by Src activation, as shown previously (4). Generation of ROS may be involved in Src activation, as the NADPH oxidase inhibitor DPI suppresses the activation of both Src and p38 (5). However, the viability of cultured cells at $24 \mathrm{~h}$ after isolation was decreased in the presence of DPI (data not shown). DPI may also inhibit enzymes that are essential for survival. In the presence of MCI-186, the cells survived and retained amylase-containing SGs. In general, primary cultures of exocrine cells rapidly lose their SGs $(14,15)$. In the present primary culture system, the cells maintained the ability to generate SGs, but the amount of amylase decreased to less than $10 \%$ of that in the cells just after isolation (about 60 units/mg protein). In this study, the addition of MCI-186 increased amylase activity as well as the number of SGs. In contrast, amylase secretion was detected in both control and MCI-186-treated cells, and the enhancement of secretion induced by $\beta$-adrenergic stimulation was comparable to that observed in the cells just after isolation. Thus, during their generation, SGs may acquire the machinery required for stimulus-dependent exocytosis.

Muscarinic stimulation induces the secretion of water and ions from salivary acinar cells. Elevation of intracellular $\mathrm{Ca}^{2+}$, mediated by the inositol triphosphate receptors $I_{3} R_{2}$ and $I P_{3} R_{3}$, is essential for this secretion (16). The increase in the F340/F380 ratio induced by addition of CCh in MCI-186-treated cells was significantly higher than the increase induced in control cells. This result suggested that fluid secretion capacity would be more

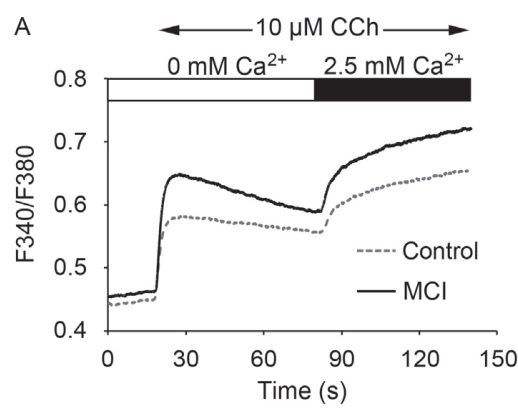

B

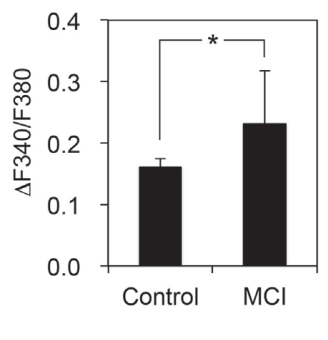

Fig. 5 Effect of MCI-186 on calcium mobilization in isolated parotid acinar cells.

(A) Cells were isolated from parotid glands in the absence and presence of MCI-186, and the freshly isolated cells were loaded with fura-2. Cells suspended in $\mathrm{Ca}^{2+}$-free buffer $\left(0 \mathrm{mM} \mathrm{Ca}{ }^{2+}\right)$ were stimulated with $10 \mu \mathrm{M}$ carbachol $(\mathrm{CCh})$, and then $2.5 \mathrm{mM}$ $\mathrm{Ca}^{2+}$ was added to the extracellular buffer. The values are shown as the ratio of the fluorescence intensities at $510 \mathrm{~nm}$ following excitation at $340 \mathrm{~nm}$ and $380 \mathrm{~nm}(\mathrm{~F} 340 / \mathrm{F} 380)$. (B) The increases in the F340/F380 ratio from the baseline to the peak response to $\mathrm{CCh}(\Delta \mathrm{F} 340 / \mathrm{F} 380)$ were compared. The response to $\mathrm{CCh}$ in the MCI-186-treated cells was significantly higher than the response in the control cells $(* P<0.05$, paired $t$-test, $n=4)$.

efficiently maintained in the presence of MCI-186. ROS production induced by irradiation and inflammatory signals has been reported to reduce $\mathrm{Ca}^{2+}$ signaling in salivary gland cells. Irradiation induces loss of STIM1, which is essential for $\mathrm{Ca}^{2+}$ influx from extracellular buffer and sustained elevation of intracellular $\mathrm{Ca}^{2+}(17)$. A phosphodiesterase inhibitor, rolipram, which suppresses ROS production, enhanced $\mathrm{CCh}$-induced $\mathrm{Ca}^{2+}$ signaling in submandibular gland cells (18). During isolation from the glands, the cells were exposed to ROS, which may depress $\mathrm{Ca}^{2+}$ signaling. The presence of MCI-186 during cell isolation may have protected the cells from ROS and increased the CCh-induced $\mathrm{Ca}^{2+}$ response.

Although ROS are reportedly involved in the impairment of salivary gland function in Sjögren's syndrome (6) and after irradiation (7), the intracellular signaling pathways responsible remain to be determined. In primary culture, ROS activated the Erk1/2-novel PKC pathway in addition to the Src-p38 pathway (19). It is expected that multiple pathways would participate in the salivary gland dysfunction caused by irradiation and inflammation. It has been shown previously that $\gamma$-irradiation induces changes in the expression patterns of intercellular adhesion molecules in vivo, and similar changes were also observed in cultured acinar cells (20). Thus, the responses triggered by cell isolation and by $\gamma$-irradiation may have common properties.

In conclusion, MCI-186 improves both the $\mathrm{Ca}^{2+}$ response and protein exocytosis in salivary acinar cells through inhibition of Src-p38 signaling. Although the 
effect of MCI-186 was small, salivary acinar cells have the potential to recover from dysfunction in vivo if the damage is not too severe. For example, the secretory function of salivary glands is temporarily impaired by $\gamma$-irradiation, but restored after several months when the radiation doses are lower (21). MCI-186 may increase the threshold for irreversible dysfunction. Further in vivo studies are needed to examine the possible clinical use of MCI-186 to prevent salivary gland dysfunction.

\section{Acknowledgments}

This study was supported by a research grant from the Research Institute of Oral Science, Nihon University School of Dentistry at Matsudo.

\section{Conflict of interest}

None.

\section{References}

1. Baum BJ (2000) Prospects for re-engineering salivary glands. Adv Dent Res 14, 84-88.

2. Fujita-Yoshigaki J, Tagashira A, Yoshigaki T, Furuyama S, Sugiya H (2005) A primary culture of parotid acinar cells retaining capacity for agonists-induced amylase secretion and generation of new secretory granules. Cell Tissue Res 320, 455-464.

3. Qi B, Fujita-Yoshigaki J, Michikawa H, Satoh K, Katsumata O, Sugiya H (2007) Differences in claudin synthesis in primary cultures of acinar cells from rat salivary gland are correlated with the specific three-dimensional organization of the cells. Cell Tissue Res 329, 59-70.

4. Fujita-Yoshigaki J, Matsuki-Fukushima M, Sugiya H (2008) Inhibition of Src and p38 MAP kinases suppresses the change of claudin expression induced on dedifferentiation of primary cultured parotid acinar cells. Am J Physiol Cell Physiol 294, C774-785.

5. Moriyama S, Yokoyama M, Katsumata-Kato O (2015) Enhancement of Src family kinase activity is essential for p38 MAP kinase-mediated dedifferentiation signal of parotid acinar cells. Int J Oral-Med Sci 14, 33-40.

6. Ryo K, Yamada H, Nakagawa Y, Tai Y, Obara K, Inoue H et al. (2006) Possible involvement of oxidative stress in salivary gland of patients with Sjögren's syndrome. Pathobiology 73, 252-260.

7. Tai Y, Inoue H, Sakurai T, Yamada H, Morito M, Ide F et al. (2009) Protective effect of lecithinized SOD on reactive oxygen species-induced xerostomia. Radiat Res 172, 331-338.

8. Watanabe T, Yuki S, Egawa M, Nishi H (1994) Protective effects of MCI-186 on cerebral ischemia: possible involvement of free radical scavenging and antioxidant actions. J Pharmacol Exp Ther 268, 1597-1604.

9. Houkin K, Nakayama N, Kamada K, Noujou T, Abe H,
Kashiwaba T (1998) Neuroprotective effect of the free radical scavenger MCI-186 in patients with cerebral infarction: clinical evaluation using magnetic resonance imaging and spectroscopy. J Stroke Cerebrovasc Dis 7, 315-322.

10. Fujita-Yoshigaki J (2011) Analysis of changes in the expression pattern of claudins using salivary acinar cells in primary culture. Methods Mol Biol 762, 245-258.

11. Nimata M, Okabe TA, Hattori M, Yuan Z, Shioji K, Kishimoto C (2005) MCI-186 (edaravone), a novel free radical scavenger, protects against acute autoimmune myocarditis in rats. Am J Physiol Heart Circ Physiol 289, H2514-2518.

12. Arii K, Kumon Y, Sugahara K, Nakatani K, Ikeda Y, Suehiro $\mathrm{T}$ et al. (2008) Edaravone inhibits collagen-induced arthritis possibly through suppression of nuclear factor-kappa B. Mol Immunol 45, 463-469.

13. Ji L, Liu Y, Zhang Y, Chang W, Gong J, Wei S et al. (2016) The antioxidant edaravone prevents cardiac dysfunction by suppressing oxidative stress in type 1 diabetic rats and in high-glucose-induced injured $\mathrm{H} 9 \mathrm{c} 2$ cardiomyoblasts. Can J Physiol Pharmacol 94, 996-1006.

14. Nicke B, Tseng MJ, Fenrich M, Logsdon CD (1999) Adenovirus-mediated gene transfer of RasN17 inhibits specific CCK actions on pancreatic acinar cells. Am J Physiol 276, G499-506.

15. Fernandez NA, Liang T, Gaisano HY (2011) Live pancreatic acinar imaging of exocytosis using syncollin-pHluorin. Am J Physiol Cell Physiol 300, C1513-1523.

16. Futatsugi A, Nakamura T, Yamada MK, Ebisui E, Nakamura K, Uchida K et al. (2005) IP3 receptor types 2 and 3 mediate exocrine secretion underlying energy metabolism. Science 309, 2232-2234.

17. Liu X, Gong B, de Souza LB, Ong HL, Subedi KP, Cheng KT et al. (2017) Radiation inhibits salivary gland function by promoting STIM1 cleavage by caspase-3 and loss of SOCE through a TRPM2-dependent pathway. Sci Signal 10, eaal4064.

18. Lee DU, Shin DM, Hong JH (2016) The regulatory role of rolipram on inflammatory mediators and cholinergic/ adrenergic stimulation-induced signals in isolated primary mouse submandibular gland cells. Mediators Inflamm 2016, 3745961.

19. Inoue D, Yokoyama M, Katsumata-Kato O (2014) Tissue injury-induced reactive oxygen species cause dysfunction of parotid acinar cells via Erk-novel PKC activation. Int J OralMed Sci 13, 6-11.

20. Yokoyama M, Narita T, Sakurai H, Katsumata-Kato O, Sugiya H, Fujita-Yoshigaki J (2017) Maintenance of claudin-3 expression and the barrier functions of intercellular junctions in parotid acinar cells via the inhibition of Src signaling. Arch Oral Biol 81, 141-150.

21. Li Y, Taylor JM, Ten Haken RK, Eisbruch A (2007) The impact of dose on parotid salivary recovery in head and neck cancer patients treated with radiation therapy. Int J Radiat Oncol Biol Phys 67, 660-669. 